\title{
Targeting Breast Cancer Using Hyaluronic Acid-Conjugated Liposomes Triggered with Ultrasound
}

\author{
Safa M. Ben Daya ${ }^{1}$, Vinod Paul ${ }^{1}$, Nahid S. Awad ${ }^{1}$, Nour M. Al Sawaftah ${ }^{1}$, \\ Mohammad H. Al Sayah², and Ghaleb A. Husseini',* \\ ${ }^{1}$ Department of Chemical Engineering, American University of Sharjah, UAE \\ ${ }^{2}$ Department of Biology, Chemistry and Environmental Sciences, American University of Sharjah, UAE
}

\begin{abstract}
The successful targeting of tumors can be achieved by conjugating targeting moieties to nanoparticles. These modifications allow nannocarriers to achieve greater targeting specificity through binding to specific receptors overexpressed on the surface of the tumor cells. In this study, pegylated liposomes encapsulating the model drug/dye calcein and conjugated to hyaluronic acid (HA) molecules were successfully synthesized, and their ability to target HA receptors overexpressed on a breast cancer cell line was investigated in vitro. Low-frequency ultrasound (LFUS), applied at three different power densities $\left(6.2,9\right.$, and $\left.10 \mathrm{~mW} / \mathrm{cm}^{2}\right)$ were used to trigger the release of the entrapped calcein. Both the control and HAconjugated liposomes showed similar release profiles. HA conjugation to the liposomes resulted in a significant increase in calcein uptake by the breast cancer cell line MDA-MB-231 known for its CD44 (HA receptor) overexpression, while such an effect was not recorded with NIH-3T3, an embryonic mouse fibroblast, with low levels of CD44 expression. The application of low LFUS showed a significant enhancement of calcein uptake by MDA-MB-231 cells from our liposome compared to calcein uptake without cell exposure to ultrasound. These findings suggest that combining HA-conjugated liposomes with ultrasound is a promising drug delivery platform in breast cancer treatment.
\end{abstract}

KEYWORDS: Hyaluronic Acid, Liposomes, Low-Frequency Ultrasound, MDA-MB-231 Cell Line, Calcein, Flow Cytometry.

\section{INTRODUCTION}

Cancer is a life-threatening disease whereby normal cells mutate, divide uncontrollably, and may in later stages of the disease invade other tissues [1]. Sadly, cancer caused the death of 9.6 million people around the world in 2018, making it the second major cause of death as per the World Health Organization. Globally, nearly 1 in 6 deaths are due to cancer [2]. Chemotherapy is one of the most common and effective treatments for cancer. However, chemotherapy is associated with many side-effects that disrupt the patient's quality of life. The lack of the targeting specificity of these therapeutic agents means that they are also cytotoxic to healthy cells [3]. A promising approach to increase bioavailability and reduce the unwanted cytotoxicity of these anti-neoplastic agents is via the use of smart drug delivery systems (SDDS) such as liposomes. Liposomes are spherical vesicles composed of phospholipid

\footnotetext{
*Author to whom correspondence should be addressed.

Email: ghusseini@aus.edu

Received: 11 December 2020

Accepted: 7 January 2021
}

bilayers [4]. Their size, amphipathic nature, and biocompatibility have rendered them amongst the best known and most promising smart drug delivery systems (DDS), in research elinies [4]. Several clinically approved liposomal formulations for cancer therapy are already used in clinics, e.g., Doxil ${ }^{\circledR}$, DaunoXome $^{\circledR}$, Onivyde ${ }^{\circledR}$, Lipo-Dox $^{\circledR}$, and others $[5,6]$.

The small size of the liposomes allows these nanocarriers to extravasate through the leaky tumor vasculature and accumulate in the diseased site; a phenomenon known as the enhanced permeability and retention (EPR) effect [7-10]. Liposomes can be modified to increase their circulation time by coating their surface with polyethylene glycol (PEG) [3, 11]. Furthermore, different types of ligands can be conjugated to these liposomes to achieve the active targeting of specific receptors overexpressed on cancer cells' surfaces, making them more cell-specific [12].

Hyaluronic acid (HA) is one of the promising targeting ligands that can be conjugated to liposomes to target different types of tumors. HA is a naturally occurring negatively charged polysaccharide formed by alternating 


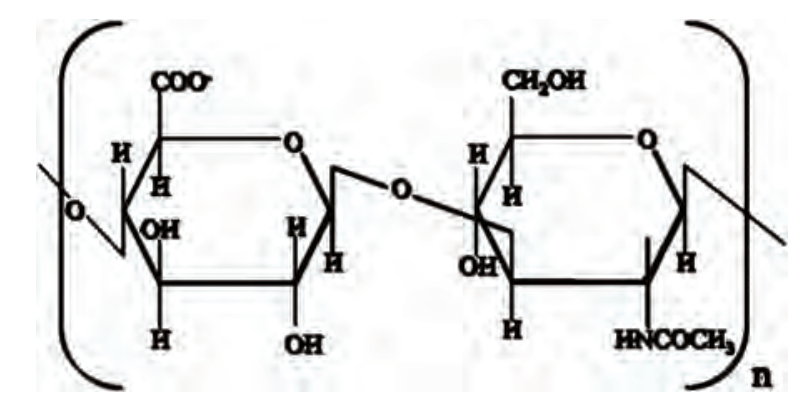

Figure 1. Chemical structure of hyaluronic acid.

$\beta-1,4$ and $\beta-1,3$ glycosidic bonds between D-glucuronic acid and D-N-acetylglucosamine [13, 14]. HA (Fig. 1) is a major constituent of the extracellular matrix (ECM) and is also found in other parts of the body such as the synovial fluid, eyes, cartilage, and skin [15]. HA has a high molecular weight $\left(10^{5}-10^{7}\right) \mathrm{Da}$ and plays important physiological roles in living organisms as a structural support and signaling molecule $[14,16]$.

HA binds to several receptors, including CD44, which is a cell membrane glycoprotein that regulates interaction, adhesion, and the migration of cells in the extar [8, 17]. CD44 is overexpressed on many tumor cells, including breast, lung, colorectal, gastric, pancreatic, renal hepatocellular, cervical cancers, and melanomas [17]. Decorating liposomes with HA has shown promise in cancer therapy. A study by Hayward et al. [18] used HA-conjugated liposomes encapsulating Doxorubicin for Glioblastoma Multiforme (GBM) The study found that HA-conjugated liposomes specifically target GBM cells over healthy brain cells due to the higher expression of CD44 receptors. Ravar et al. [19] showed that HA conjugation to paclitaxel (PTX)-loaded liposomes led to higher uptake by the breast cancer cell lines 4T1 and T47D and thus, enhanced the agent's cytotoxic activity in comparison to free PTX and non-targeted PTX loaded liposomes. HA-conjugation also resulted in high tumor accumulation and antitumor activity when tested on 4T1 tumor-bearing mice.

Following the accumulation of the liposomes in the tumor site, several internal and external stimuli can be employed to trigger the release of the encapsulated drug, such as $\mathrm{pH}$, temperature, enzymes, and ultrasound [20]. Combining active targeting with a triggering mechanism results in reducing drug toxicity and increasing therapeutic efficacy. Recently, ultrasound has gained special attention in inducing drug release from liposomes in a spatially and temporally controlled manner [21-23]. Ultrasound is the transmission of pressure waves through a medium at frequencies above $20,000 \mathrm{~Hz}$ [24]. The wide use of ultrasound-based medical diagnostic and therapeutic techniques is justified because ultrasound is safe, costeffective, and can be easily focused on specific targeted tissues [25]. Therapeutic ultrasound can produce thermal effects (through heating the tissue) or non-thermal effects (also known as "mechanical effects"). While the thermal effects are generally associated with high-intensity focused ultrasound (HIFU) in the continuous mode, the mechanical effects of ultrasound, also known as the acoustic cavitation, can be generated using pulsed low-frequency ultrasound [23]. Ultrasonic waves at a given frequency and intensity can induce several biophysical effects on exposed tissue, including cavitation, sonoporation, and hyperthermia [26]. Cavitation is the formation of gas bubbles due to pressure changes [24]. As the pressure wave passes, the resulting bubbles oscillate without collapsing (stable cavitation) or expand rapidly and eventually collapse (transient cavitation) [25, 27]. Cavitation can induce the formation of tiny transient or permanent pores in the walls of blood vessels surrounding the tumor (sonoporation), resulting in a significant enhancement of the extravascular delivery of therapeutics to the cancer cells [26, 27].

In this study, HA-conjugated liposomes are used to target breast cancer cells overexpressing HA receptors (CD44). The role of low frequency ultrasomnd in triggering drug release and enhancing drug uptake will be explored in vitro.

\section{MATERIALS AND METHODS Materials}

DPPC (Dipalmitoylphosphatidyl choline) and DSPEPEG(2000)- $\mathrm{NH}_{2}$ (1,2-distearoyl-sn-glycero-3-phosphoethanolamine-N [amino(polyethylene glycol)-2000]) were obtained from Avanti Polar Lipids Inc. AL, USA), Calcein disodium salt, cholesterol, chloroform, Sephadex G-100, hyaluronic acid (Mw: 170 KD), $\mathrm{N}$-Ethyl-N'-(3-dimethylaminopropyl)carbodiimide (EDC), N-Hydroxysuccinimide (NHS), 2-(N-Morpholino) ethanesulfonic acid hemisodium salt (MES), and Triton ${ }^{\mathrm{TM}}$ X-100 were obtained from Sigma Aldrich Chemie GmbH (Munich, Germany, supplied by Labco LLC. Dubai, UAE). MDA-MB-231 and NIH-3T3 cell lines were obtained from the European Collection of Authenticated Cell Cultures (ECACC).

\section{Liposome Preparation}

Liposomes encapsulating calcein were prepared using the modified lipid-film-hydration method reported by Lasch et al. [28]. DPPC, DSPE-PEG (2000)- $\mathrm{NH}_{2}$ and cholesterol were dissolved in chloroform in a round bottom flask at molar ratios of 30:65:5, respectively. Chloroform was evaporated using a rotatory evaporator under vacuum at $50{ }^{\circ} \mathrm{C}$ for 15 min until a dry, thin lipid film was formed around the interior of the round bottom flask. Two $\mathrm{ml}$ of a $20 \mathrm{mM}$ calcein solution $(\mathrm{pH} 7.4)$ was used to hydrate the lipid film for $50 \mathrm{~min}$ at $60{ }^{\circ} \mathrm{C}$ using a rotatory evaporator. The suspension was sonicated for $2 \mathrm{~min}$ at $60{ }^{\circ} \mathrm{C}$ using a sonication bath to obtain unilamellar vesicles. The liposomes were then extruded through a membrane filter of pore size $200 \mathrm{~nm}$ and filtered using a Sephadex G-100 
column equilibrated with phosphate-buffered saline (PBS buffer).

\section{HA-Conjugated Liposomes Preparation}

HA-conjugated liposomes were prepared using a modified method reported by Hayward et al. [29]. Hyalurenic aeid. was dissolved in an MES buffer $(\mathrm{pH} \sim 4.5)$ to a final concentration of $4 \mathrm{mg} / \mathrm{ml}$. EDC was added to activate the carboxylic acid in $\mathrm{HA}$ and $\mathrm{N}$ hydrexysuccinimide (NHS) was also added to enhance HA conjugation to the liposomes (Fig. 2). The activated HA solution was combined with the liposome solution and the $\mathrm{pH}$ was adjusted to 8.6. The reaction mixture was incubated for 6 hours at room temperature with gentle mixing to mediate the amide bond formation. The separation of the resulting HA-liposomes from excess reagents was achieved by eentrifuging at $32750 \mathrm{RCF}$ for 1 hour at $4{ }^{\circ} \mathrm{C}$, followed by resuspending the pellets in PBS buffer.

\section{Liposomes Characterization}

DynaPro NanoStar DLS (Wyatt Technology Corp., Santa Barbara, California, USA) was used to determine both the mean size and polydispersity of the prepared liposomes. Also, the zeta potentials of the conjugated and non-conjugated liposomes were measured using Malvern Zetasizer Nanomachine (Worcestershire, UK).

\section{Phospholipid Concentration Quantification}

The phospholipid concentration in the prepared liposomes was determined colorimetrically using the Stewart Assay [30]. Fifty $\mu$ l of the liposome, were dried using a rotatory evaporator and dissolved in $1 \mathrm{ml}$ of chloroform. This solution was then sonicated and transferred to a Pyrex centrifuge tube. An ammonium ferrothiocyanate solution $(2 \mathrm{ml})$ was added and the tube was then sonicated for $20 \mathrm{~s}$ followed by centrifugation for $10 \mathrm{~min}$ at $1000 \mathrm{rpm}$. The bottom layer containing the colored complex was collected and its absorbance at $485 \mathrm{~nm}$ was measured using a UVVis Spectrophotometer (Thermo Scientific Genesys 10S). A calibration curve was prepared using DPPC $(0.1 \mathrm{mg} / \mathrm{ml})$ to determine the exact concentration of the phospholipids in the prepared liposomes.

\section{Measuring the Power Density of the Ultrasonic Probe}

Ultrasonic waves are caused by the vibration of the probe's surface; the density of the power produced is proportional to the amplitude of the probe's vibration. To measure the acoustic power density produced by the probe at different amplitudes, we used a hydrophone (Bruel and Kjaer 8103, Nærum, Denmark). The hydrophone detects the pressure variations in the medium created by the ultrasonic waves and converts these events into voltage signals. These voltage signals are then fed to a digital storage oscilloscope (Tektronix TDS 2002B) to visualize and a computer for further processing using the MATLAB software.

A water bath was used to conduct the measurements and the hydrophone was placed at a depth of $3 \mathrm{~cm}$ from the tip of the probe (Fig. 3). While maintaining a constant distance in the ' $Z$ ' direction (the distance between the hydrophone and the probe), the hydrophone was raster scanned through an area of $7 \mathrm{~cm} \times 5 \mathrm{~cm}$ (in $X-Y$ direction) around the probe and the signals were picked up at every interval of $1 \mathrm{~cm}$. The measurements were made at probe amplitudes of 20, 25 and $30 \%$ (as shown on the display of the machine). The measured voltage signals were then converted into acoustic pressure in Pascal using the equation:

$$
P=\frac{\operatorname{Vrms}(V)}{\text { Voltage Sensitivity }(\mu V / P a)}
$$

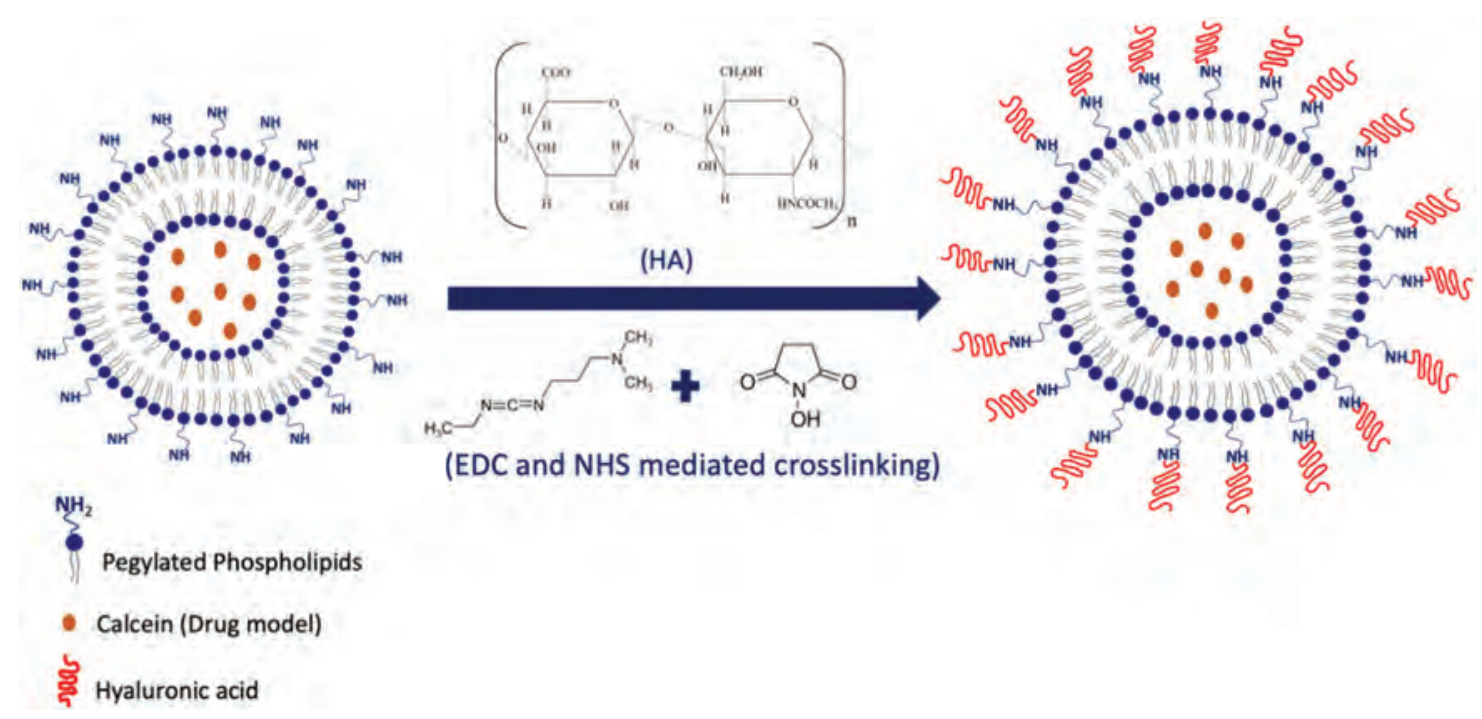

Figure 2. Conjugation of hyaluronic acid (HA) to liposomes via EDC and NHS facilitated amide bond formation. 


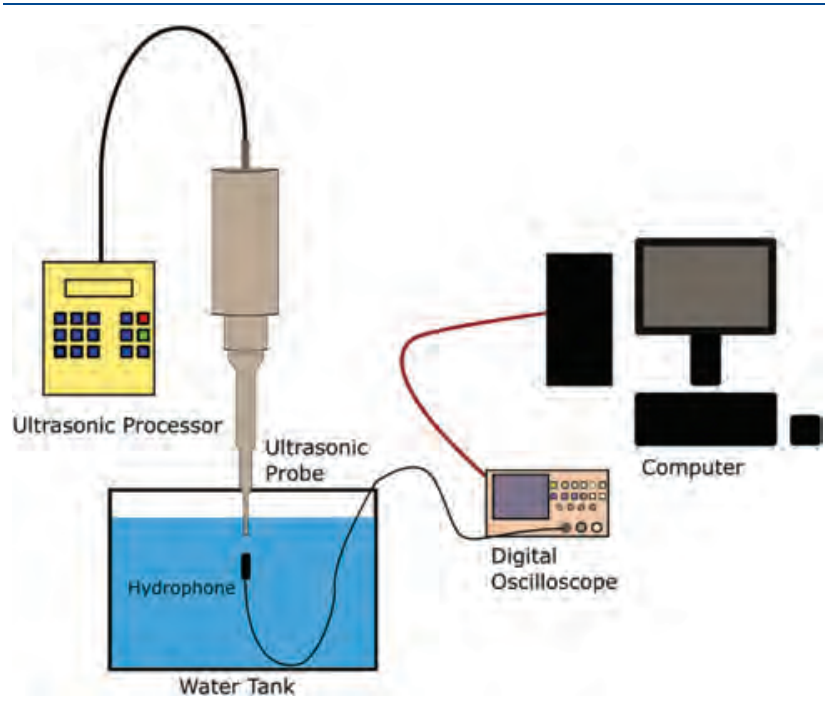

Figure 3. A schematic diagram showing the setup of the experiment.

The value of the hydrophone voltage sensitivity was provided by the hydrophone manufacturer as $30 \mu \mathrm{V} / \mathrm{Pa}$.

And the ultrasound power density ' $I$ ' in Watt $/ \mathrm{cm}^{2}$ is given by the equation.

$$
\text { Power Density, } I=\frac{P^{2}}{Z}
$$

Where ' $Z$ ' is the acoustic impedance of the medium $\left(1.48 \times 10^{6} \mathrm{Kg} \cdot \mathrm{m}^{-2} \cdot \mathrm{S}^{-1}\right.$, the impedance of water $)$ and ' $P$ ' is the pressure measured in Pascals.

\section{LFUS Triggering of Calcein Release}

The release of the encapsulated calcein was triggered using a 20-kHz low-frequency ultrasonic probe (model VCX750, Sonics and Materials Inc., Newtown, CT) and monitored by changes in fluorescence using a QuantaMaster QM 30 Spectrofluorometer (Photon Technology International, Edison, NJ, USA). Calcein is a fluorescent molecule with excitation and emission wavelengths of $495 \mathrm{~nm}$ and $515 \mathrm{~nm}$, respectively. The samples were prepared by diluting $75 \mu \mathrm{l}$ of liposomes in $3 \mathrm{ml}$ of PBS buffer. The initial fluorescence intensity $t_{\theta_{2}}$ was measured for 20 seconds before ultrasound sonication in a pulsed mode (20 seconds "on" and 10 seconds "off"). Different power densities were tested. The liposomes were then lysed and $100 \%$ release was achieved using Triton X-100. The percentage of calcein release at a given time was then calculated according to the following equation.

$$
\% \text { Release }=\frac{F-F_{0}}{F_{T x 100}-F_{0}} \times 100
$$

Where $F$ is the fluorescence intensity at the time $(t), F_{0}$ is the initial fluorescence intensity before US, and $F_{T X-100}$ is the maximum fluorescence achieved after lysing the liposomes.

\section{Cell Cultures}

MDA-MB-231 (HA-positive human breast adenocarcinoma) and NIH-3T3 (HA-negative embryonic fibroblast) cell lines were cultured in RPMI and DMEM media, respectively, supplemented with $10 \%$ heat-inactivated fetal bovine serum (FBS) and $1 \%$ penicillin-streptomycin (Sigma Aldrich, St. Louis, MO, USA). The cultures were kept inside an incubator $\left(37{ }^{\circ} \mathrm{C}, 5 \% \mathrm{CO}_{2}\right)$. Twenty-four hours before the uptake studies, exponentially growing cells were harvested with $3 \mathrm{ml}$ of trypsin-EDTA $(0.25 \%)$ and $6 \times 10^{5}$ cells were seeded in 6 -well plates to reach confluency at the time of the experiment.

\section{Flow Cytometry Study}

For the cellular uptake studies, $\mathrm{CD} 44^{+}$cells (MDA-MB231) and CD44- ${ }^{-}$cells (NIH-3T3) were seeded into 6-well plates at a concentration of $2 \times 10^{5}$ cells $/ \mathrm{ml}(3 \mathrm{ml}$ to each well). After overnight incubation, the cell culture media from the wells were discarded and fresh serum-free media containing the control and HA-conjugated liposome were added. The cell culture plates were then incubated for 2 hours before sonication in a $40-\mathrm{kHz}$ US bath (Branson 3510-DTH Ultrasonic bath) for $5 \mathrm{~min}$ and were returned to the same incubation conditions for 2 additional hours. Once the incubation period was over, the cells were trypsinized using trypsin-EDTA. The detached cell samples were then washed twice with PBS and transferred to 5-ml tubes. The amount of calcein internalized inside the cells was then analyzed using flow cytometry (Beckman Coulter FC500, USA) by measuring the calcein fluorescence intensity excited by a blue laser at $488 \mathrm{~nm}$ and emited at $525 \mathrm{~nm}$ (FL1). At least three independent assays were performed for each treatment.

\section{Statistical Analysis}

Results were reported as average \pm standard deviation (SD). One-way ANOVA tests were used to compare the size and zeta potential of the control and HA-conjugated liposomes, while two-factor ANOVA tests were employed to analyze LFUS release results. Both types of ANOVA tests were based on the assumption that both populations have similar variances, and two values were statistically different if $p<0.05$ and if $F<F_{\text {critical }}$ (unless otherwise stated).

\section{RESULTS}

\section{Liposomes Characterization and Attachment Confirmation}

The phospholipids content of the prepared liposomes was estimated using the Stewart assay. Both the control and HA-conjugated liposomes contained similar phospholipids' concentration showing values of $9.43 \pm 0.66 \mathrm{mg} / \mathrm{ml}$ and $10.03 \pm 0.93 \mathrm{mg} / \mathrm{ml}$, respectively $(P=0.318)$. HA is a large, negatively charged molecule. Therefore, changes 
Table I. Size (radius), percentage polydispersity (Pd\%), and zeta potential of control and HA-conjugated liposomes. The results are the average of 3-replicates.

\begin{tabular}{lccc}
\hline Liposomes & Radius $(\mathrm{nm})$ & $\mathrm{Pd} \%$ & Zeta potential $(\mathrm{mV})$ \\
\hline Control liposomes & $83.6 \pm 1.1$ & $14.1 \pm 1.7$ & $-6.59 \pm 0.38$ \\
$\begin{array}{l}\text { HA-conjugated } \\
\text { liposomes }\end{array}$ & $107.6 \pm 2.2$ & $16.8 \pm 1.5$ & $-22.50 \pm 2.27$ \\
\begin{tabular}{l}
$P$-value \\
\hline
\end{tabular} & 0.0002 & & 0.0006 \\
\hline
\end{tabular}

in size and zeta potential of the liposomes were used as a criterion to assess the conjugation of HA to the liposomal surface. Table I summarizes the average size, polydispersity, and zeta potential of three batches of liposomes with their respective standard deviations. Results show that HA conjugation produced a significant increase in the size and negative zeta potentials of the liposomes $(P<0.05)$ due to the presence of repeated carboxylic negative residues, which are major components of HA molecules.

\section{Ultrasound Power Densities Measurements}

The hydrophone was used to measure the power density produced by the LFUS processor at amplitudes of $20 \%, 25 \%$, and $30 \%$. The depth of the hydrophone inside the water bath was kept constant, but the distance from the probe was varied to create a small 2-D acoustic map that shows the effect of changing the distance of the hydrophone (from the probe) on the power density. The results showed that the highest intensity recorded was when the hydrophone was the closest to the tip of the probe at all three tested amplitudes $(20 \%, 25 \%$, and $30 \%)$. The highest power density recorded was $6.2 \mathrm{~mW} / \mathrm{cm}^{2}$, $9 \mathrm{~mW} / \mathrm{cm}^{2}$ and $10 \mathrm{~mW} / \mathrm{cm}^{2}$ at the $20 \%, 25 \%$ and $30 \%$ displayed amplitudes, respectively. Figure 4(A) shows the measured power intensity values of an area at a distance of $3 \mathrm{~cm}$ from the tip of the probe at a probe amplitude of $30 \%$.

\section{Drug Release Kinetics from Liposomes Triggered with LFUS}

A detailed investigation of LFUS-triggered release of calcein from both control and HA-conjugated liposomes was conducted using a fluorometer. The fluorescence level of the released calcein was measured and used to calculate the cumulative fraction release (CFR) given by the equation.

$$
\mathrm{CFR}=\frac{I_{t}-I_{0}}{I_{F}-I_{0}}
$$

Figure 5 shows the normalized-average release profiles of the control (non-targeted) and HA-conjugated liposomes (three batches of liposomes each). Both types of liposomes were sonosensitive to 20-kHz LFUS applied in a pulsed mode with $20 \mathrm{sec}$ "on" and $10 \mathrm{sec}$ "off" for $3 \mathrm{~min}$ and $20 \mathrm{sec}$ with increasing power densities (6.2, 9 , and $10 \mathrm{~mW} / \mathrm{cm}^{2}$ ). Calcein was released from both the

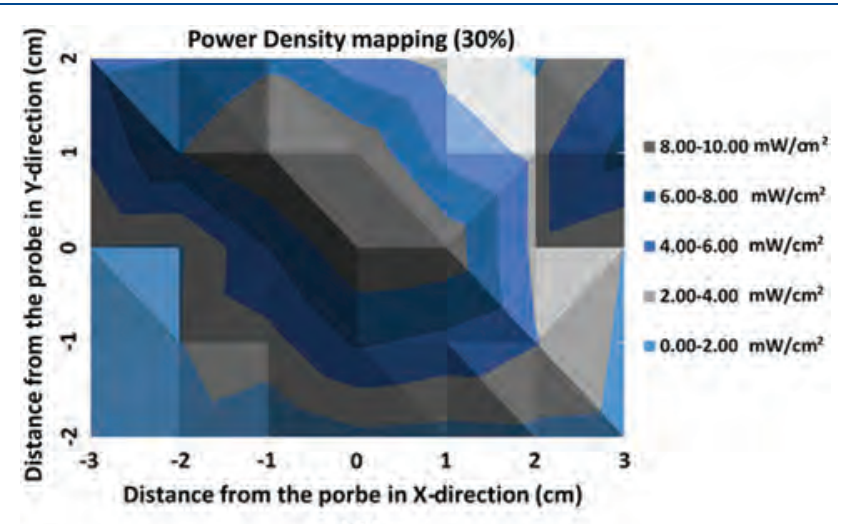

(A)

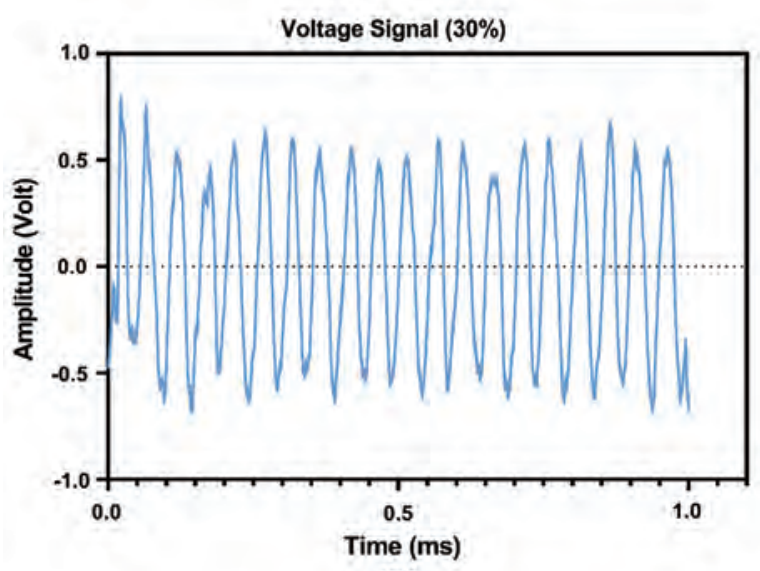

(B)

Figure 4. Power intensity values at various distances from the probe at $30 \%$ amplitude $(\mathrm{A})$ and the voltage signal measured by the digital oscilloscope of the highest power density recorded at $30 \%(B)$.

control and HA-conjugated liposomes during the "on" portion of the pulse and plateaued during the "off" period. We noticed a clear overlap of the calcein release profile between both types of liposomes, indicating that HA conjugation did not affect the nanocarriers' sonosensitivity to LFUS. Calcein release increased as we increased the acoustic power density. Additionally, lysing the liposomes with Triton X-100 revealed that both types of liposomes released most of their cargo following $3 \mathrm{~min}$ of pulsed sonication. To further confirm that calcein release is due to ultrasound triggering, calcein release from nonsonicated liposomes was also studied. As seen in Figure 5, No increase in fluorescence was observed when liposomes were not subjected to ultrasound; hence our nanoparticles remained intact and undamaged with no measurable calcein release until Triton X-100 was added. Also, no change in the size of the liposomes was reported following pulsed sonication.

A more detailed comparison between the percentages of drug release during the first three pulses at each power density was performed. The findings, presented in 


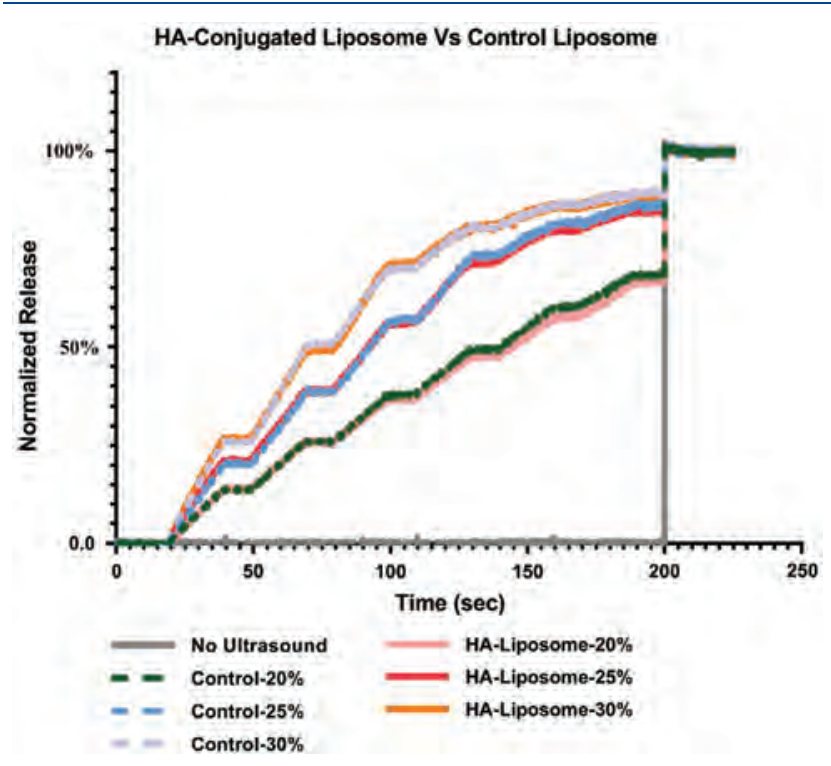

Figure 5. Percent calcein release from control and HAconjugated liposomes following sonication at 20-kHz LFUS for $3 \mathrm{~min}$ and $20 \mathrm{sec}$ at three power densities $\left(6.2 \mathrm{~mW} / \mathrm{cm}^{2}\right.$, $\left.9 \mathrm{~mW} / \mathrm{cm}^{2}, 10 \mathrm{~mW} / \mathrm{cm}^{2}\right)$. Non-sonicated liposomes showed no measurable calcein release. Triton $\mathrm{X}-100$ was added to release all encapsulate contents hence simulating $100 \%$ release. Results are averages of three batches ( 3 replicates each).

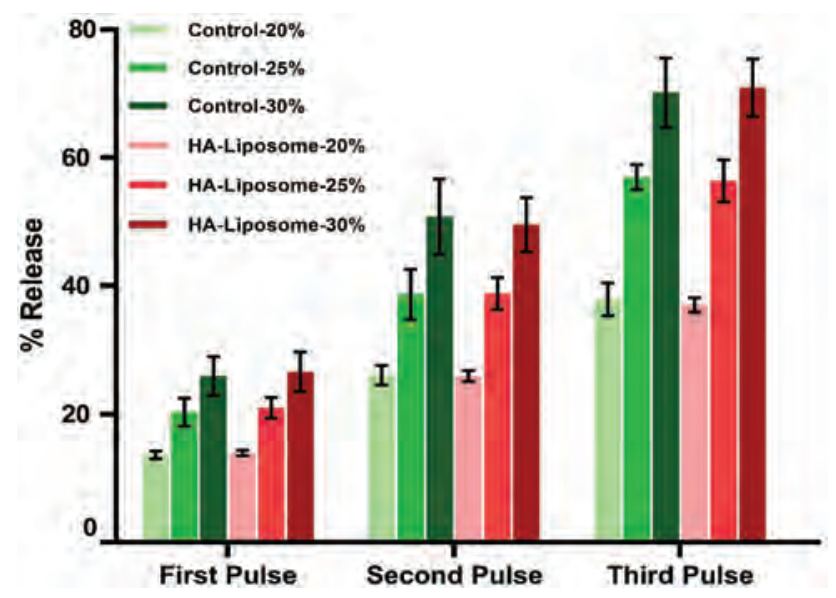

Figure 6. Percent release of calcein encapsulated inside the control and HA-conjugated liposomes following the first three acoustic pulses at different power densities $20 \%\left(6.2 \mathrm{~mW} / \mathrm{cm}^{2}\right)$, $25 \%\left(9 \mathrm{~mW} / \mathrm{cm}^{2}\right), 30 \%\left(10 \mathrm{~mW} / \mathrm{cm}^{2}\right)$. Results are the average of three liposome batches (3 replicates each).

Figure 6 , showed that the $\%$ drug release increased as the power density increased with no statistically significant difference between the control and HA-conjugated liposomes in all the power densities tested here $(p>0.05)$.

\section{Enhanced Cellular Uptake of HA-Conjugated Liposomes In Vitro}

Flow cytometry was used to determine calcein uptake by MDA-MB-231 (HA-positive human breast adenocarcinoma) and NIH-3T3 (HA-negative embryonic fibroblast) breast cancer cells. Figure 7 shows the cellular uptake of calcein following the incubation of both cell lines with either the control or HA-conjugated liposomes for 4 hours at $37^{\circ} \mathrm{C}$. The average fluorescent intensity of HAconjugated liposomes was $18346 \pm 874.01$ in the MDAMB-231 breast cancer cell line with a significant increase of $260 \%\left(p\right.$-value $\left.=3.99 \times 10^{-6}\right)$ compared to the average fluorescent intensity of the control liposomes (3532 \pm 24.23). The sonication of HA-conjugated liposomes with LFUS, using a $40-\mathrm{kHz}$ water bath, further increased their average fluorescent intensity by $100 \%$, i.e., $43324 \pm 309.51$ $\left(p\right.$-value $\left.=2.67 \times 10^{-8}\right)$. Overall, the average fluorescent intensity of the LFUS treated HA-conjugated liposomes was $640 \%$ higher than that of the control liposomes $\left(p\right.$-value $\left.=4.45 \times 10^{-5}\right)$.

Further experiments were conducted to confirm that the significant increase in calcein uptake by the MDA-MB231 cells was due to the binding, and subsequent internalization of the HA-conjugated liposomes, as a result of the overexpression of the HA-receptors on the surface of these cells. The same liposomal formulations were incubated for 4 hours with embryonic fibroblast cells (NIH-3T3) known for their low expression level of the HA-receptors. Only a slight increase in the average fluorescent intensity (28\%) was recorded with HA-conjugated liposomes $(6129 \pm 130.52)$ compared to the control liposomes $(5140 \pm 293.51)$. While this small increase is significant $(p$-value $=0.0004)$, it is 9 times lower than that measured with the targeted liposomes $(260 \%)$. These findings indicate that the low expression of HA-receptors on the surface of the NIH-3T3 cells resulted in reducing the targeting efficiency of the HA-conjugated liposomes and the subsequent low cellular uptake of the encapsulated calcein.

\section{DISCUSSION}

The present study is part of the efforts to reduce the side effects and enhance the efficacy of chemotherapeutic drugs. This can be achieved via encapsulating antineoplastic agents inside targeted liposomes, followed by applying ultrasound as a triggering mechanism. Thus, providing safe, efficient, and controlled drug release at the diseased site. moiety because it is an abundant component of the exa cellelar matrix and is directly involved in cellular proliferation and migration, and hence plays an important role in cancer development and progression [31]. CD44 are specific glycoprotein receptors that bind to HA molecules and are overexpressed on the surface of many tumors. This study was designed to explore whether HA-conjugated liposomes can be used to target breast cancer by binding to the CD44 receptors. Once bound to the receptors, we investigated releasing more of the encapsulated drug in a spatially and temporally controlled manner. The liposomal phospholipid bilayer is similar to that of biological 

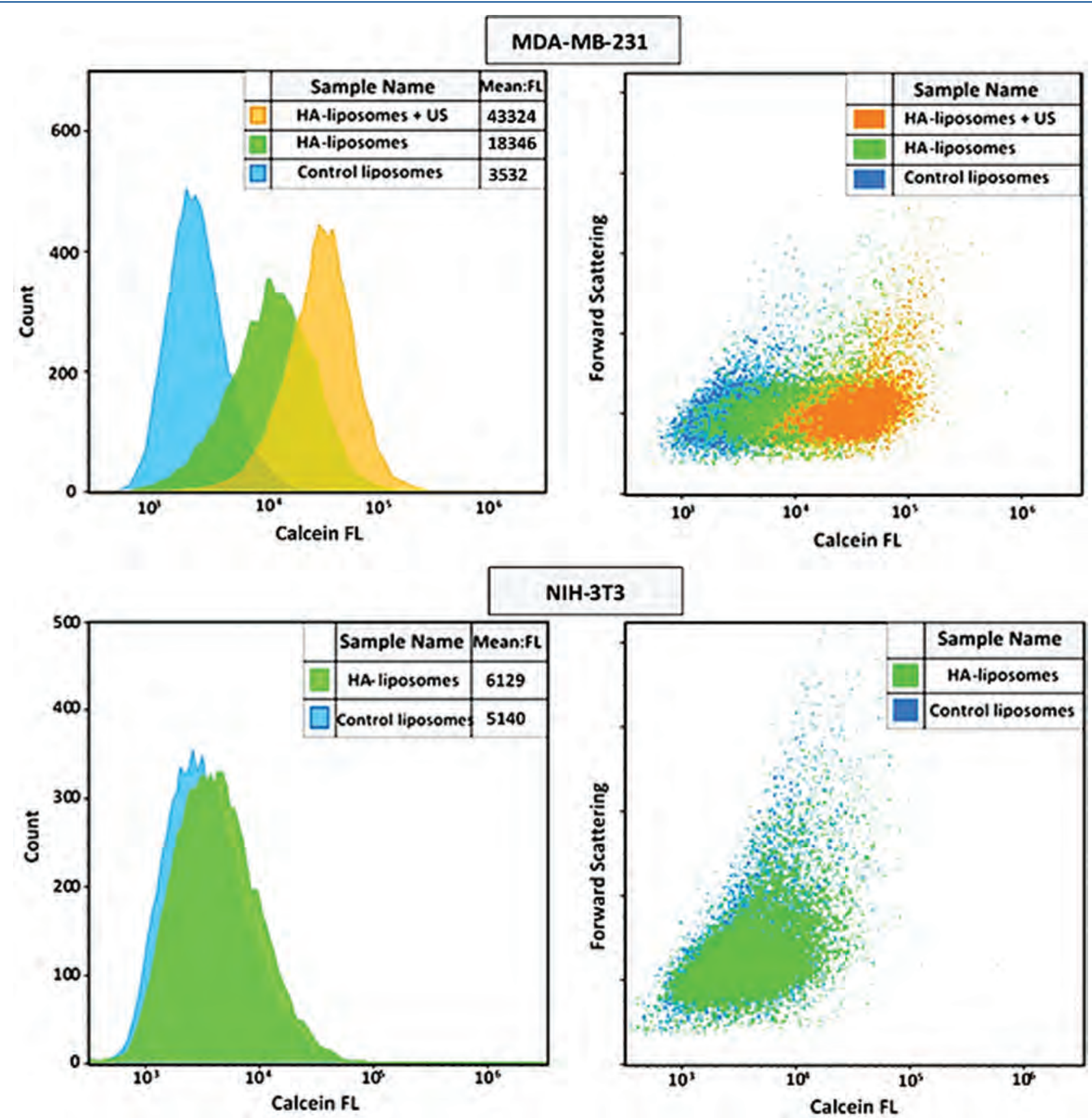

Figure 7. Calcein uptake by the breast cancer cell lines (MDA-MB-231) is significantly higher from the HA-conjugated liposomes compared to the control liposomes (top). Sonication with LFUS (40 kHz for $5 \mathrm{~min}$ ) resulted in further enhancement of calcein uptake. No significant increase in calcein uptake was recorded when HA-conjugated liposomes were incubated with NIH-3T3 cells compared to the control liposomes (bottom). Results are the average of three liposome batches (3 replicates each).

membranes; thus, ultrasound application increases their permeability and, subsequently, triggers the release of the loaded drug in a controlled manner [32].

In this study, HA conjugation to the liposomal lipid bilayer was achieved through the post insertion method, and resulted in a significant increase in the average radius size from $83.6 \mathrm{~nm}$ to $107.6 \mathrm{~nm}$ which indicates that HA molecules were conjugated to the outer layer of the liposomes. The size of HA-conjugated liposomes was slightly over the recommended size of nanoparticles used in drug delivery $(<200 \mathrm{~nm})$ [19]. The recommended size range is essential to allow the nanoparticles to extravasate through the pores formed in the leaky tumor vasculature due to the EPR (enhanced permeability and retention) effect. Although a nanoparticle size of $12.5-150 \mathrm{~nm}$ is recommended, the EPR effect generally operates in the range of $100 \mathrm{~nm}$ to $400 \mathrm{~nm}$ [33]. The presence of carboxyl groups in the HA molecules (Fig. 1) resulted in 
increasing the negativity of the surface charge, which also suggests that HA molecules are (at least partially) covering the surface of the liposomes. Previous studies have also used a similar method to confirm HA conjugation to the liposomes [18, 34-38]. The polydispersity percentage $(\mathrm{Pd} \%)$ remained under $20 \%$ following HA conjugation. HA molecules are hydrophilic and, like PEG molecules, attract abundant quantities of water, thus reducing aggregation [39]. HA-conjugated liposomes had a similar structure to the liposomes and showed no aggregation as reported previously by Arpicco et al. [40] and more recently by Song et al. [41].

In this study, LFUS triggered calcein release from both the control and HA-conjugated liposomes. The release was detected during the "on" pulse period but stopped during the "off" period of ultrasound, which confirms that ultrasound is the sole release trigger rather than other factors such as lipid oxidation or damage. Two of the three important parameters of LFUS (frequency and pulse duration) were kept constant; we only varied the power density. Cavitation is likely to be the mechanism behind the enhanced calcein release. Despite an increase in temperature from $25^{\circ} \mathrm{C}$ to $31{ }^{\circ} \mathrm{C}$ following the third acoustic pulse, this temperature rise is still below the transition temperature of the phospholipid used to prepare the liposomes (DPPC), i.e., $41.3{ }^{\circ} \mathrm{C}$. When loaded liposomes are heated to their transition temperature, membrane lipids are transformed from the solid-state to the liquid-state [42]. Thus, causing membrane defects and drug release. Since calcein release was recorded at temperatures lower than the transition temperature, we posit that mechanical effects rather than thermal effects are the main mechanisms behind the observed release. Previous studies have shown that transient cavitation, which generates sonic waves following bubbles collapse, was the main release triggering mechanism from liposomes exposed to LFUS [43, 44]. In our experiments, no change in the size of the liposomes was reported following the first three pulses of LFUS. This suggests that the observed calcein release is due to pore formation on the liposomal membrane (pore-mediated release) rather than the complete destruction of these nanocarriers, as previously reported by Evjen et al. [45]. When exposed to LFUS (20-kHz), both the control and HA-conjugated liposomes showed a similar level of sonosensitivity with overlapping release profiles following pulsed sonication. These findings confirm that the presence of HA molecules on the liposomal surfaces had no interference with the LFUS effect on triggering drug release despite the relatively large size of the HA molecules.

The ability of the prepared HA-conjugated liposomes to bind to CD44 receptors, overexpressed on the surface of the breast cancer cell line MDA-MB-231, under in vitro conditions was tested. Embryonic fibroblast cells (NIH-3T3) were used as a negative control for the differential binding of HA-conjugated and control liposomes.
The significant increase in calcein uptake by MDA-MB231 cells from the HA-conjugated liposomes compared to the control liposomes could be attributed to the binding of these targeted nanocapsules to their specific receptors. NIH-3T3 are known for their very low expression of CD44 [46], which explains the low calcein uptake of targeted liposomes. This is in agreement with previous studies that reported an enhanced drug uptake from HAconjugated liposomes compared to non-targeted liposomes as a result of HA-CD44 interaction [34, 47].

We then tested the effect of LFUS on the cellular uptake of HA-conjugated liposomes and found that LFUS (at $40-\mathrm{kHz}$ ) enhanced cellular uptake of both the control and HA-conjugated liposomes. The exposure to LFUS creates pores on the membranes of the cells (sonoporation), thus enhancing the level of cellular uptake of calcein, which is consistent with the amount of the formed pores as reported by previous studies $[48,49]$. Cell membrane poration, coupled with the acoustic release of the agent from our ligandtargeted liposomes, increased calcein's accumulation in the HA-positive cell line by $640 \%$. This study is conducted in vitro in the absence of blood vessels and blood circulation; thus it is considered a first step or a proof of concept. Future work including a detailed in vivo analysis using breast tumor models is essential to develop further understanding of the ultimate therapeutic efficacy of this platform.

\section{CONCLUSION}

In this study, HA-conjugated liposomes loaded with calcein were successfully synthesized with a mean radius of $107.7 \mathrm{~nm}$ and a negative surface charge. In vitro, lowfrequency ultrasound triggered calcein release more release as the power density increased. Both the control and targeted liposomes released most of their cargo following 3 minutes of pulsed sonication; HA conjugation did not affect liposome sensitivity to LFUS. Additionally, HA-conjugated liposomes exhibited improved cellular uptake based on CD44-mediated endocytosis. These findings suggest that combining targeted drug delivery with an ultrasound-triggering mechanism can significantly increase the therapeutic index of drugs and minimize their toxic side effects.

\section{Conflicts of Interest}

There are no conflicts to declare.

Acknowledgments: The authors would like to acknowledge funding from AUS Faculty Research Grants (FRGs, eFRGs and BBRI grants), the Patient's Friends Committee-Sharjah, AlJalila Foundation, Al Qasimi Foundation, the Technology Innovation Pioneer-Healthcare (TIP) Program, Takamul and the Dana Gas Endowed Chair for Chemical Engineering. 


\section{REFERENCES}

1. National Cancer Institute, What Is Cancer? 2015. (https://www. cancer.gov/about-cancer/understanding/what-is-cancer\#celldifferences).

2. World Health Organization, Cancer. (https://www.who.int/healthtopics/cancer\#tab=tab_1).

3. Steichen, S.D., Caldorera-Moore, M. and Peppas, N.A., 2013 A review of current nanoparticle and targeting moieties for the delivery of cancer therapeutics. European Journal of Pharmaceutical Sciences, 48(3), pp.416-427.

4. Akbarzadeh, A., Rezaei-Sadabady, R., Davaran, S., Joo, S.W., Zarghami, N., Hanifehpour, Y., Samiei, M., Kouhi, M. and NejatiKoshki, K., 2013. Liposome: Classification, preparation, and applications. Nanoscale Research Letters, 8(1), p.102.

5. Beltrán-Gracia, E., López-Camacho, A., Higuera-Ciapara, I., Velázquez-Fernández, J.B. and Vallejo-Cardona, A.A., 2019. Nanomedicine review: Clinical developments in liposomal applications. Cancer Nanotechnology, 10(1), p.11.

6. Bulbake, U., Doppalapudi, S., Kommineni, N. and Khan, W., 2017. Liposomal formulations in clinical use: An updated review. Pharmaceutics, 9(2), p.12.

7. Ruttala, H.B., Ramasamy, T., Madeshwaran, T., Hiep, T.T., Kandasamy, U., Oh, K.T., Choi, H.G., Yong, C.S. and Kim, J.O., 2018. Emerging potential of stimulus-responsive nanosized anticancer drug delivery systems for systemic applications. Archives of Pharmacal Research, 41(2), pp.111-129.

8. Alavi, M. and Hamidi, M., 2019. Passive and active targeting in cancer therapy by liposomes and lipid nanoparticles. Drug Metabolism and Personalized Therapy, 34(1).

9. Yingchoncharoen, P., Kalinowski, D.S. and Richardson, D.R., 2016. Lipid-based drug delivery systems in cancer therapy: What is available and what is yet to come. Pharmacological Reviews, 68(3), pp.701-787.

10. Riaz, M.K., Riaz, M.A., Zhang, X., Lin, C., Wong, K.H., Chen, X., Zhang, G., Lu, A. and Yang, Z., 2018. Surface functionalization and targeting strategies of liposomes in solid tumor therapy: A review. International Journal of Molecular Sciences, 19(1), p.195.

11. Han, N.K., Shin, D.H., Kim, J.S., Weon, K.Y., Jang, C.Y. and Kim, J.S., 2016. Hyaluronan-conjugated liposomes encapsulating gemcitabine for breast cancer stem cells. International Journal of Nanomedicine, 11, p.1413.

12. Yu, B., Tai, H.C., Xue, W., Lee, L.J. and Lee, R.J., 2010. Mol. Membr. Biol., 27, pp.286-298.

13. Necas, J., Bartosikova, L., Brauner, P. and Kolar, J., 2008 Hyaluronic acid (hyaluronan): A review. Veterinarni Medicina, 53(8), pp.397-411.

14. Dicker, K.T., Gurski, L.A., Pradhan-Bhatt, S., Witt, R.L., FarachCarson, M.C. and Jia, X., 2014. Hyaluronan: A simple polysaccharide with diverse biological functions. Acta Biomaterialia, 10(4), pp.1558-1570.

15. Fakhari, A. and Berkland, C., 2013. Applications and emerging trends of hyaluronic acid in tissue engineering, as a dermal filler and in osteoarthritis treatment. Acta Biomaterialia, 9(7), pp.7081-7092.

16. Dhanusha, B., 2015. Hyaluronic acid and its applications. Res. Rev. J. Med. Heal. Sci., 4.

17. Qhattal, H.S.S. and Liu, X., 2011. Characterization of CD44mediated cancer cell uptake and intracellular distribution of hyaluronan-grafted liposomes. Molecular Pharmaceutics, 8(4), pp.1233-1246.

18. Hayward, S.L., Wilson, C.L. and Kidambi, S., 2016. Hyaluronic acid-conjugated liposome nanoparticles for targeted delivery to CD44 overexpressing glioblastoma cells. Oncotarget, 7(23), p.34158.

19. Ravar, F., Saadat, E., Gholami, M., Dehghankelishadi, P., Mahdavi, M., Azami, S. and Dorkoosh, F.A., 2016. Hyaluronic acid-coated liposomes for targeted delivery of paclitaxel, in-vitro characterization and in-vivo evaluation. Journal of Controlled Release, 229, pp.10-22.

20. Tayo, L.L., 2017. Stimuli-responsive nanocarriers for intracellular delivery. Biophysical Reviews, 9(6), pp.931-940.

21. Awad, N.S., Paul, V., Al-Sayah, M.H. and Husseini, G.A., 2019. Ultrasonically controlled albumin-conjugated liposomes for breast cancer therapy. Artificial Cells, Nanomedicine, and Biotechnology, 47(1), pp.705-714.

22. Awad, N.S., Paul, V., Mahmoud, M.S., Al Sawaftah, N.M., Kawak, P.S., Al Sayah, M.H. and Husseini, G.A., 2019. Effect of pegylation and targeting moieties on the ultrasound-mediated drug release from liposomes. ACS Biomaterials Science \& Engineering, 6(1), pp.48-57.

23. Ahmed, S.E., Awad, N., Paul, V., Moussa, H.G. and Husseini, G.A., 2018. Improving the efficacy of anticancer drugs via encapsulation and acoustic release. Current Topics in Medicinal Chemistry, 18(10), pp.857-880.

24. Pitt, W.G., Husseini, G.A. and Staples, B.J., 2004. Ultrasonic drug delivery-A general review. Expert Opinion on Drug Delivery, 1(1), pp.37-56.

25. Chowdhury, S.M., Lee, T. and Willmann, J.K., 2017. Ultrasoundguided drug delivery in cancer. Ultrasonography, 36(3), p.171.

26. Tharkar, P., Varanasi, R., Wong, W.S.F., Jin, C. and Chrzanowski, W., 2019. Nano-enhanced drug delivery and therapeutic ultrasound for cancer treatment and beyond. Frontiers in Bioengineering and Biotechnology, 7, p.324.

27. Frenkel, V., 2008. Ultrasound mediated delivery of drugs and genes to solid tumors. Advanced Drug Delivery Reviews, 60(10), pp.11931208.

28. Torchilin, P.V., Torchilin, V.P., Torchilin, V. and Weissig, V., eds., 2003. Liposomes: A Practical Approach (No. 264). Oxford University Press.

29. Hayward, S.L., Francis, D.M., Kholmatov, P. and Kidambi, S., 2016. Targeted delivery of MicroRNA125a-5p by engineered lipid nanoparticles for the treatment of HER2 positive metastatic breast cancer. Journal of Biomedical Nanotechnology, 12(3), pp.554-568.

30. Stewart, J.C.M., 1980. Colorimetric determination of phospholipids with ammonium ferrothiocyanate. Analytical Biochemistry, 104(1), pp.10-14.

31. Chen, C., Zhao, S., Karnad, A. and Freeman, J.W., 2018. The biology and role of CD44 in cancer progression: Therapeutic implications. Journal of Hematology \& Oncology, 11(1), p.64.

32. Pitt, W.G., Husseini, G.A., Roeder, B.L., Dickinson, D.J., Warden, D.R., Hartley, J.M. and Jones, P.W., 2011. Preliminary results of combining low frequency low intensity ultrasound and liposomal drug delivery to treat tumors in rats. Journal of Nanoscience and Nanotechnology, 11(3), pp.1866-1870.

33. Bagchi, M., Moriyama, H. and Shahidi, F., 2012. BioNanotechnology: A Revolution in Food, Biomedical and Health Sciences. John Wiley \& Sons.

34. Pandolfi, L., Frangipane, V., Bocca, C., Marengo, A., Tarro Genta, E., Bozzini, S., Morosini, M., D'Amato, M., Vitulo, S., Monti, M. and Comolli, G., 2019. Hyaluronic acid-decorated liposomes as innovative targeted delivery system for lung fibrotic cells. Molecules, 24(18), p.3291.

35. Yu, J.S., Shin, D.H. and Kim, J.S., 2020. Repurposing of fluvastatin as an anticancer agent against breast cancer stem cells via encapsulation in a hyaluronan-conjugated liposome. Pharmaceutics, 12(12), p.1133.

36. Marengo, A., Forciniti, S., Dando, I., Dalla Pozza, E., Stella, B., Tsapis, N., Yagoubi, N., Fanelli, G., Fattal, E., Heeschen, C. and Palmieri, M., 2019. Pancreatic cancer stem cell proliferation is strongly inhibited by diethyldithiocarbamate-copper complex loaded into hyaluronic acid decorated liposomes. Biochimica et Biophysica Acta (BBA)-General Subjects, 1863(1), pp.61-72.

37. Lin, Q., Mao, K.L., Tian, F.R., Yang, J.J., Chen, P.P., Xu, J., Fan, Z.L., Zhao, Y.P., Li, W.F., Zheng, L. and Zhao, Y.Z., 2016. Brain 
tumor-targeted delivery and therapy by focused ultrasound introduced doxorubicin-loaded cationic liposomes. Cancer Chemotherapy and Pharmacology, 77(2), pp.269-280.

38. Qian, Y., Liang, X., Yang, J., Zhao, C., Nie, W., Liu, L., Yi, T., Jiang, Y., Geng, J., Zhao, X. and Wei, X., 2018. Hyaluronan reduces cationic liposome-induced toxicity and enhances the antitumor effect of targeted gene delivery in mice. ACS Applied Materials \& Interfaces, 10(38), pp.32006-32016.

39. Snetkov, P., Zakharova, K., Morozkina, S., Olekhnovich, R. and Uspenskaya, M., 2020. Hyaluronic acid: The influence of molecular weight on structural, physical, physico-chemical, and degradable properties of biopolymer. Polymers, 12(8), p. 1800 .

40. Marengo, A., Forciniti, S., Dando, I., Dalla Pozza, E., Stella, B., Tsapis, N., Yagoubi, N., Fanelli, G., Fattal, E., Heeschen, C. and Palmieri, M., 2019. Pancreatic cancer stem cell proliferation is strongly inhibited by diethyldithiocarbamate-copper complex loaded into hyaluronic acid decorated liposomes. Biochimica et Biophysica Acta (BBA)-General Subjects, 1863(1), pp.61-72.

41. Song, M., Liang, Y., Li, K., Zhang, J., Zhang, N., Tian, B. and Han, J., 2019. Hyaluronic acid modified liposomes for targeted delivery of doxorubicin and paclitaxel to CD44 overexpressing tumor cells with improved dual-drugs synergistic effect. Journal of Drug Delivery Science and Technology, 53, p.101179.

42. de Matos, M.B., Beztsinna, N., Heyder, C., Fens, M.H., Mastrobattista, E., Schiffelers, R.M., Leneweit, G. and Kok, R.J., 2018. Thermosensitive liposomes for triggered release of cytotoxic proteins. European Journal of Pharmaceutics and Biopharmaceutics, 132, pp.211-221.
43. Schroeder, A., Avnir, Y., Weisman, S., Najajreh, Y., Gabizon, A., Talmon, Y., Kost, J. and Barenholz, Y., 2007. Controlling liposomal drug release with low frequency ultrasound: Mechanism and feasibility. Langmuir, 23(7), pp.4019-4025.

44. Lin, H.Y. and Thomas, J.L., 2004. Factors affecting responsivity of unilamellar liposomes to $20 \mathrm{kHz}$ ultrasound. Langmuir, 20(15), pp.6100-6106.

45. Evjen, T.J., Hupfeld, S., Barnert, S., Fossheim, S., Schubert, R. and Brandl, M., 2013. Physicochemical characterization of liposomes after ultrasound exposure-mechanisms of drug release. Journal of Pharmaceutical and Biomedical Analysis, 78, pp.118-122.

46. Tzircotis, G., Thorne, R.F. and Isacke, C.M., 2005. Chemotaxis towards hyaluronan is dependent on CD44 expression and modulated by cell type variation in CD44-hyaluronan binding. Journal of Cell Science, 118(21), pp.5119-5128.

47. Park, J.H., Cho, H.J., Yoon, H.Y., Yoon, I.S., Ko, S.H., Shim, J.S., Cho, J.H., Park, J.H., Kim, K., Kwon, I.C. and Kim, D.D., 2014. Hyaluronic acid derivative-coated nanohybrid liposomes for cancer imaging and drug delivery. Journal of Controlled Release, 174, pp.98-108.

48. Theek, B., Baues, M., Ojha, T., Möckel, D., Veettil, S.K., Steitz, J., van Bloois, L., Storm, G., Kiessling, F. and Lammers, T., 2016. Sonoporation enhances liposome accumulation and penetration in tumors with low EPR. Journal of Controlled Release, 231, pp.77-85.

49. Jelenc, J., Miklavčič, D. and Lebar, A.M., 2013. Low-Frequency Ultrasound In Vitro: Experimental System and Ultrasound-Induced Changes of Cell Morphology. 2013 36th International Convention on Information and Communication Technology, Electronics and Microelectronics (MIPRO), IEEE, pp.340-344. 